\title{
Constraints on the Accuracy of Photometric Redshifts Derived from BLAST and Herschel/SPIRE Sub-mm Surveys
}

\author{
Itziar Aretxaga*, David. H. Hughes*, Edward Chapin* and Enrique Gaztañaga* \\ *INAOE, Aptdo. Postal 51 y 216, 72000 Puebla, Mexico
}

\begin{abstract}
More than 150 galaxies have been detected in blank-field millimetre and sub-millimetre surveys. However the redshift distribution of sub-mm galaxies remains uncertain due to the difficulty in identifying their optical-IR counterparts, and subsequently obtaining their spectroscopic emission-line redshifts. In this paper we discuss results from a Monte-Carlo analysis of the accuracy with which one can determine redshifts from photometric measurements at sub-millimetre-FIR wavelengths. The analysis takes into account the dispersion in colours introduced by including galaxies with a distribution of SEDs, and by including photometric and absolute calibration errors associated with real observations. We present examples of the probability distribution of redshifts for individual galaxies detected in the future BLAST and Herschel/SPIRE surveys. We show that the combination of BLAST and $850 \mu \mathrm{m}$ observations constrain the photometric redshifts with sufficient accuracy to pursue a program of spectroscopic follow-up with the 100m GBT.
\end{abstract}

\section{DESCRIPTION OF THE TECHNIQUE AND RESULTS}

Determining the density of star formation as a function of redshift is the primary science objective of the Balloonborne Large Aperture Submillimetre Telescope (BLAST, [1]) and other sub- $\mathrm{mm} / \mathrm{mm}$ facilities. Using Monte-Carlo simulations that take into account realistic photometric and absolute calibration errors for future BLAST surveys, we show that it is possible to determine redshifts from BLAST data (at 250,350 and $500 \mu \mathrm{m}$ ) with a $1 \sigma$ average precision of $\Delta z \sim \pm 0.6$. A similar level of redshift accuracy is found for simulated observations with the Herschel/SPIRE camera which will operate at identical wavelengths to BLAST.

The power of this simple technique to derive redshifts arises from the unique ability of BLAST and SPIRE observations to bracket the ubiquitous rest-frame FIR peak (at $\sim 60-150 \mu \mathrm{m}$ ) in the spectral energy distribution (SED) of high-redshift $(1 \leq z \leq 4)$ galaxies undergoing a significant amount of star formation.

To determine the accuracy of this method we have generated mock catalogues of galaxies between $z=0$ and $z=6$ using an evolving $60 \mu \mathrm{m}$ luminosity function [2] that reproduces the observed $850 \mu \mathrm{m}$ number counts. The sub-mm flux densities, and colours, of these mock galaxies are calculated from SEDs selected at random from a library of template starburst galaxies, ULIRGs and AGN (Fig.1). Observational noise is then added to the intrinsic fluxes: $1 \sigma$ photometric errors of 5 and $2.5 \mathrm{mJy}$ for the BLAST and Herschel/SPIRE observations respectively, and in both cases an absolute calibration error of $7 \%$.

We are therefore able to determine the photometric redshift probability distribution for any galaxy detected in BLAST surveys by comparing its measured BLAST colours with the complete distribution of simulated colours and redshifts of galaxies in the mock catalogue (see Fig.2).

It is instructive to illustrate the discrepancy between the photometric redshifts determined from the colours of galaxies detected in our simulated BLAST (or SPIRE) surveys and their true mock catalogue redshifts. Figure 3 shows that over the entire redshift range of our simulations, $0 \leq z \leq 6$, the $1 \sigma$ error in the photometric redshifts, $\Delta z$, derived from detections in 3 BLAST filters is \pm 0.6 . However beyond $z \sim 4$ the BLAST data alone systematically underestimate the redshifts, confusing $z \sim 4.5$ galaxies with $z \sim 2.5$ galaxies for example, as the longest wavelength BLAST filter $(500 \mu \mathrm{m})$ moves short-ward of the rest-frame FIR peak.

A significant improvement in the derived photometric redshifts occurs when the BLAST (or SPIRE) observations are complemented with data from a longer-wavelength survey (e.g. ground-based $850 \mu \mathrm{m}$ SCUBA data). This extension to the wavelength coverage $(250-850 \mu \mathrm{m})$ ensures that the photometric redshifts are uniformly distributed about the 


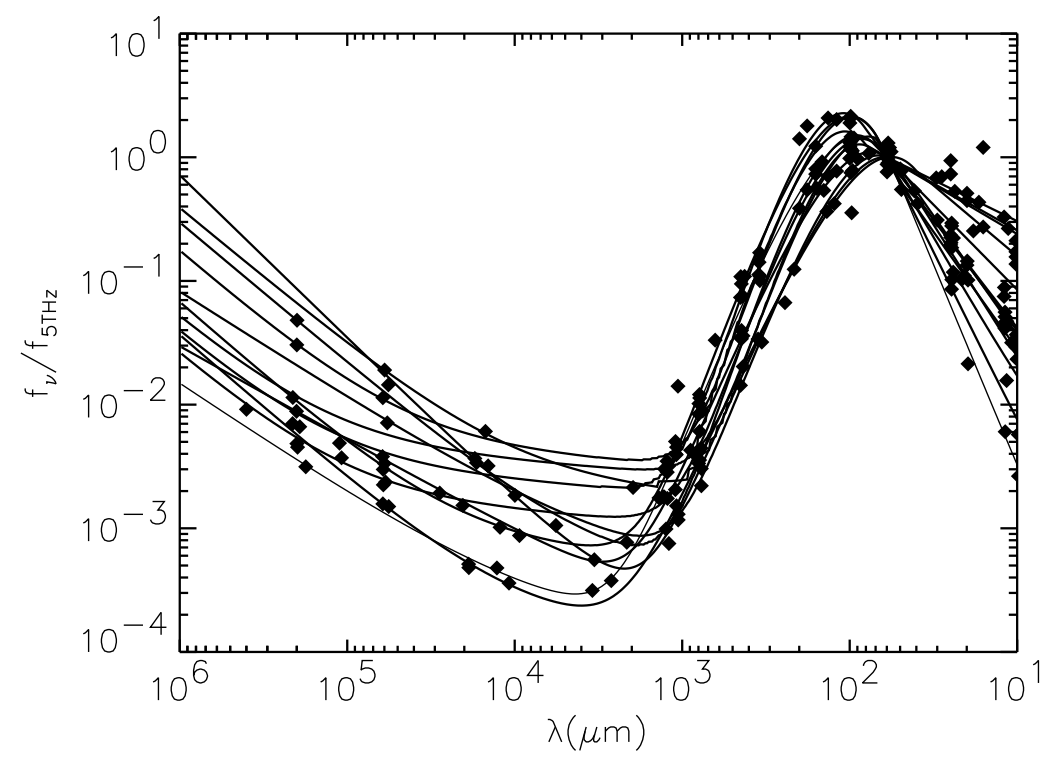

FIGURE 1. Rest-frame spectral energy distributions of 13 starburst galaxies, ULIRGs and AGN, normalized at $60 \mu \mathrm{m}$. Lines represent the best fit models to the SEDs and include contributions from non-thermal synchrotron emission, free-free and grey-body thermal emission.

$z=z_{\text {phot }}$ regression line over the entire range $0<z<6$, with an improved average error of $\Delta z \sim \pm 0.4$ (Fig. 3). This method therefore continues to provide un-biased estimates of photometric redshifts for the most distant galaxies. Furthermore, an increased sensitivity in the observations (with SPIRE for instance) naturally translates into an increased accuracy of the redshift distributions.

\section{OPPORTUNITIES FOR SPECTROSCOPIC FOLLOW-UP}

Redshift accuracies can be further improved with heterodyne follow-up observations. We plan to use the recently commissioned 100m GBT to search for low-J CO molecular-line transitions. For example, for those BLAST and Herschel/SPIRE galaxies with photometric redshifts in the range $z \sim 4.0-4.8$, which have $1 \sigma$ accuracy of $\Delta z \sim \pm 0.4$ when combined with $850 \mu$ m observations, we can search for $\mathrm{CO}(1-0)$ in the K-band $(18.0-26.5 \mathrm{GHz})$. The addition of a future GBT receiver operating at $26-40 \mathrm{GHz}$ will extend the $\mathrm{CO}(1-0)$ search to galaxies at $2.3 \lesssim z \lesssim 4.8$. Similarly, a proposed $68-116 \mathrm{GHz}$ receiver, will detect both the $\mathrm{CO}(1-0)$ and $\mathrm{CO}(2-1)$ transitions of the low- $z$ population $(z \lesssim 2)$.

To conclude, our simulations demonstrate that, within the next few years, large-area BLAST surveys will produce a catalogue of $\gtrsim 5000$ high- $z$ galaxies for which sub-mm photometric redshifts with an accuracy $\Delta z \sim \pm 0.4-0.6$ can be determined. Follow-up observations with GBT receivers will provide definitive molecular-line redshifts and dynamical-mass estimates. The combination of BLAST and GBT provide a powerful combination to break the redshift deadlock that hinders our ability to understand the evolution and nature of the sub-mm starburst galaxy population.

Acknowledgments: This work has been partly supported by CONACyT grants 32143-E and 32180-E.

\section{REFERENCES}

1. Devlin, M. et al. 2001, in Deep Millimetre Surveys: Implications for Galaxy Formation and Evolution, eds. J.Lowenthal, D.H.Hughes, World Scientific

2. Saunders, W. et al. 1990, MNRAS, 424,318 

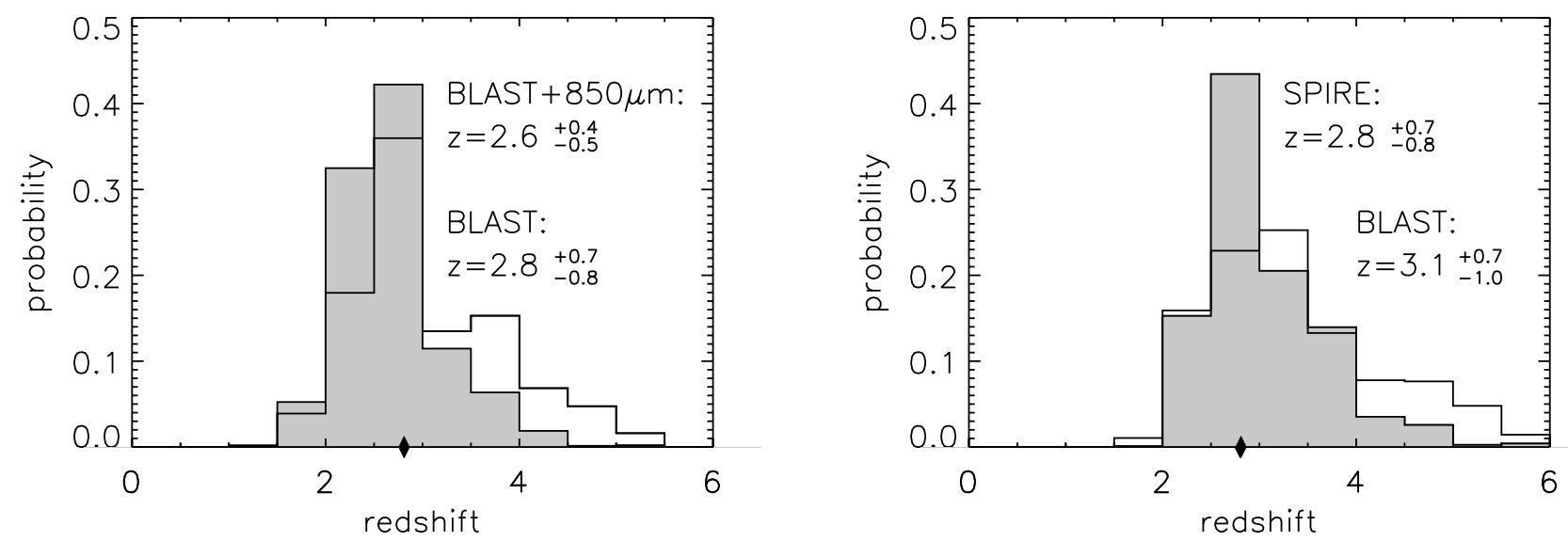

FIGURE 2. Left panel: Redshift probability distributions of a $4 \times 10^{12} \mathrm{~L} \odot$ galaxy at $z=2.8$, detected at 250,350 and $500 \mu \mathrm{m}$ with BLAST, and also at $850 \mu \mathrm{m}$ in a ground-based deep survey. The intrinsic colours of this galaxy are determined from a scaled SED of NGC 1614: $S_{250 \mu \mathrm{m}}=17 \mathrm{mJy}, S_{350 \mu \mathrm{m}}=23 \mathrm{mJy}, S_{500 \mu \mathrm{m}}=27 \mathrm{mJy}, S_{850 \mu \mathrm{m}}=5 \mathrm{mJy}$. The unshaded and shaded distributions have been calculated from BLAST detections and from BLAST $+850 \mu \mathrm{m}$ detections, respectively. The black diamond marks the true redshift of the mock galaxy. Photometric redshift determinations within a $68 \%$ confidence level are indicated within the panel. Right panel: A comparison of the redshift distributions for a $3 \times 10^{12} \mathrm{~L}_{\odot}$ galaxy at $z=2.8$ determined from SPIRE and the less sensitive BLAST observations. The colours of this galaxy are similar to NGC 2992: $S_{250 \mu \mathrm{m}}=18 \mathrm{mJy}, S_{350 \mu \mathrm{m}}=28 \mathrm{mJy}, S_{500 \mu \mathrm{m}}=23 \mathrm{mJy}$, $S_{850 \mu \mathrm{m}}=7 \mathrm{mJy}$. The galaxy was detected simultaneously in the 3 SPIRE passbands, but only at 350 and 500 $\mu \mathrm{m}$ with BLAST. The unshaded and shaded histograms correspond to the use of BLAST and SPIRE detections, respectively.
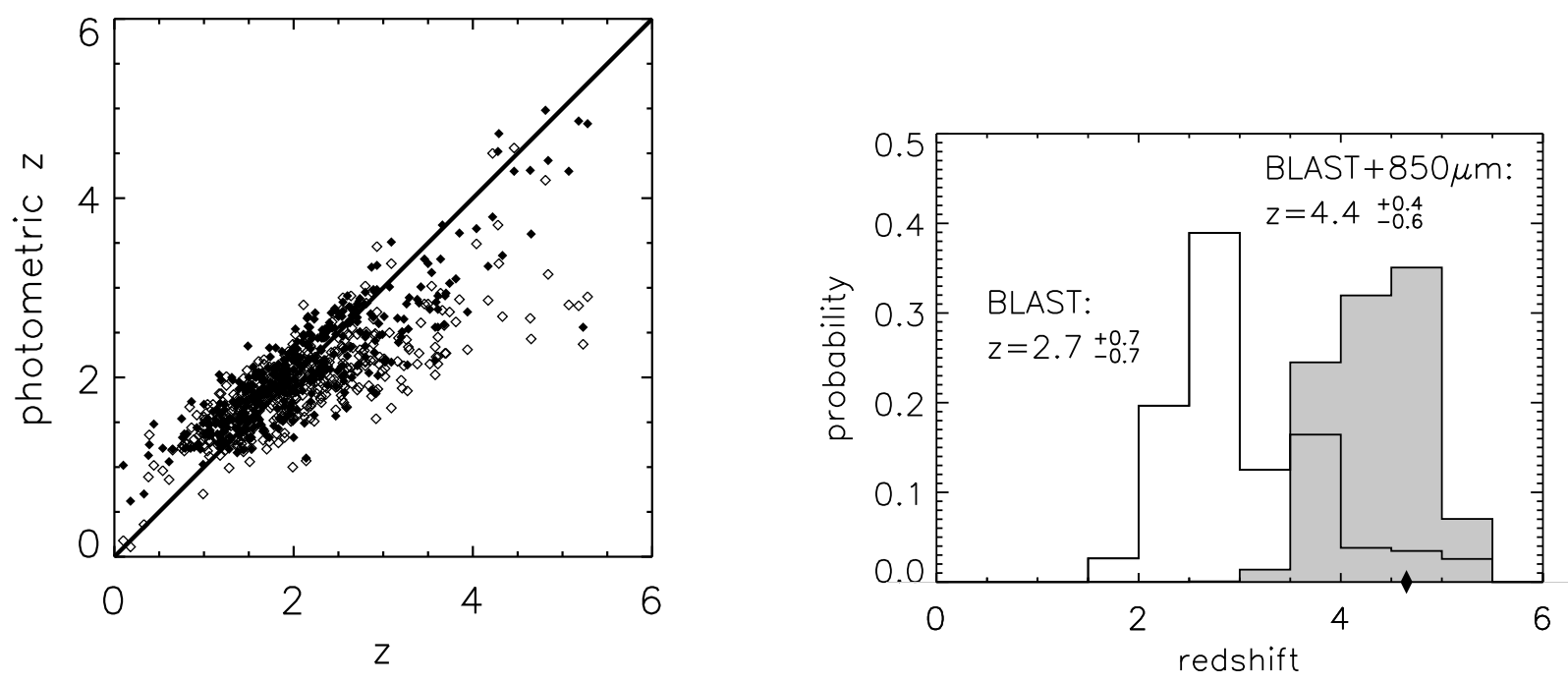

FIGURE 3. Left panel: Photometric redshift vs. true redshift relationship for 400 galaxies simultaneously detected at 250 , 350 and $500 \mu \mathrm{m}$ in 1 sq. degree. Open symbols show the relationship inferred using only BLAST data to derive the redshifts. Filled symbols show the relationship when the redshifts are estimated using colours based on BLAST and complementary 850 $\mu \mathrm{m}$ detections from a ground-based survey. The addition of $850 \mu \mathrm{m}$ measurements significantly increases the accuracy at $z \gtrsim 4$, since at these redshifts the BLAST filters sample the rest-frame mid-IR to FIR $(\sim 35-100 \mu \mathrm{m})$. The longer wavelength data are required to bracket the rest-frame FIR peak, which provides the diagnostic power for the photometric redshift technique discussed in this paper. Right panel: Example of the correction attained at $z>4$ when $850 \mu \mathrm{m}$ observations are included in the photometric redshift analysis. In particular, these are redshift distributions of a $1 \times 10^{13} \mathrm{~L} \odot$ galaxy at $z=4.65$, with observed fluxes $S_{250 \mu \mathrm{m}}=18 \mathrm{mJy}$, $S_{350 \mu \mathrm{m}}=29 \mathrm{mJy}, S_{500 \mu \mathrm{m}}=27 \mathrm{mJy}, S_{850 \mu \mathrm{m}}=19.5 \mathrm{mJy}$. 